\title{
Transformation of dynamical fluctuation into coherent energy
}

\author{
Tsuyoshi Hondou \\ Department of Physics, Tohoku University \\ Sendai 980-77, Japan \\ (e-mail: hondou@cmpt01.phys.tohoku.ac.jp)
}

\begin{abstract}
Studies of noise-induced motions are showing that coherent energy can be extracted from some kinds of noise in a periodic ratchet. Recently, energetics of Langevin dynamics is formulated by Sekimoto [J.Phys.Soc.Jpn, 661234 (1997)], which can be applied to ratchet systems described by Fokker-Planck equation. In this paper, we derive an energetics of ratchet systems that can be applied to dynamical-noise-induced motion in a static potential. Analytical efficiency of the energy transformation is derived for the dynamical noise in an overdumping limit of the system. Comparison between analytical and numerical studies is performed for chaotic noise.
\end{abstract}

How efficient can noise be transformed into coherent energy? Recent advance of the studies of molecular motors 1] wakes us up to the basic problem. A famous trial to this problem can be found in the lecture note by Feynman [5], where he derived the efficiency of energy transformation in a system, what is called Feynman's ratchet, where multistability of the ratchet makes energy transformation available. Recently, Sekimoto formulated energetics of Langevin equation [6]. He applied it to the Feynman's ratchet and found an essential dissipation mechanism that was lost in Feynman's discussion.

The formulation of energetics of Langevin equation [6] is so generic that it may be analytically applicable to the system if it can be described by Fokker-Planck equation [7], where an additive noise should be statistically simple. On the other hand, noise that breaks detailed balance of the applied system may often have statistically complex structure. A good example of such noise is chaos, where the fluctuation is generated by dynamical process. However the effect of such dynamical noise on the multistable system cannot be analyzed by Fokker-Planck equation for its un-negligible higher order time-correlation function. Thus, the energetics for these dynamical systems has not been constructed yet, to my knowledge.

In this paper, we try to construct a theory that describes the transformation of the dynamical noise into coherent energy by a periodic ratchet. First, we introduce a prototype system where the energy transformation is available. We estimate in the next step the input and the output energy and define the efficiency of the energy transformation. By introducing a dynamical noise that is generated by a map, we derive an analytical expression of the efficiency of energy transformation. For checking our method, we compare the analytical result with numerical one using a chaotic map as a dynamical noise. With discussion for future studies, we conclude this paper.

Let us discuss a ratchet system where a particle moves in one dimensional periodic potential with resistance proportional to its velocity and an additive noise. This system is described by a generalized Langevin equation,

$$
m \frac{d^{2} x}{d t^{2}}=-\gamma \frac{d x}{d t}+\xi(t)-\frac{\partial V(x)}{\partial x},
$$

where $m, \gamma, \xi$, are a mass of the particle, a resistance coefficient and an additive noise respectively. $V(x)$ is a periodic potential of which multistability makes it possible to transform noise into coherent energy. We divide the noise term, $\xi(t)$, between $\xi_{\mathrm{T}}(t)$ and $\xi_{\mathrm{d}}(t): \xi(t)=\xi_{\mathrm{T}}(t)+\xi_{\mathrm{d}}(t)$, where $\left\langle\xi_{\mathrm{T}}(t) \xi_{\mathrm{T}}(s)\right\rangle=2 \gamma k_{\mathrm{B}} T \delta(t-s)$. The system does not move coherently if $\xi_{\mathrm{d}}=0$, because the system then holds "detailed balance". In such a case, the energy transformation is not available, which is the result of the second law of thermodynamics. Because the effect of $\xi_{\mathrm{d}}(t)$ causes finite drift of the system and therefore energy transformation, we consider zero temperature limit. To discuss the energy transformation, we add a mechanical load to the periodic potential:

$$
V(x)=V_{0}(x)+V_{l}(x),
$$

where $V_{0}(x)$ is a periodic potential and $V_{l}(x)$ is a potential by which a load to the system is described. We set $V_{l}(x)=l x$ for simplicity. As a prototype, we adopt a piecewise linear potential with parity symmetry as the periodic potential, $V_{0}(x): V_{0}(x)=h-$ $(h / L)|x(\bmod (2 L))-L|$ for $x \geq 0, V_{0}(-x) \equiv V_{0}(x)$, where $L$ is a half width of the period of the potential and $h$ is a height of the potential barrier.

To discuss the energetics of the present system, we define the input energy, $R$, from the noise to the system:

$$
R=\int_{x_{i}\left(t_{i}\right)}^{x_{f}\left(t_{f}\right)} \xi(t) d x(t) .
$$

We also define a mechanical energy, $E_{l}$, that the system acquires coherently in the fluctuating process:

$$
E_{l}=V\left(x_{f}, t_{f}\right)-V\left(x_{i}, t_{i}\right) .
$$

Then, the efficiency of the energy transformation, $\epsilon_{t}$, between an initial state $\left(t_{i}, x_{i}\right)$ and a final state $\left(t_{f}, x_{f}\right)$ is defined:

$$
\epsilon_{t}=\frac{E_{l}}{R}
$$


The obtained coherent energy, $E_{l}$, is globally proportional both to the strength of the load, $l$, and to the displacement of the system, $\Delta x$ :

$$
E_{l}=l \cdot \Delta x
$$

In general, we have to solve Eq.(1) to evaluate the efficiency of the energy transformation, Eq.(5). If the dominant noise was thermal, $\xi(t) \sim \xi_{\mathrm{T}}$, Eq.(1) could be reduced to Fokker-Planck equation, where average current could be obtained analytically. However, when the noise is dynamic, $\xi(t) \sim \xi_{d}(t)$, the reduction is not successful, because the time-correlation function of the noise can not be truncated by second order. Recently, it was found that an average current induced by chaotic noise could be estimated analytically by focusing one's attention on the deterministic nature of the time series [4. Therefore we can proceed to discuss the energetics induced by dynamical noise. We introduce dynamical noise that is described by a map:

$$
\xi(t)=\Sigma_{i=-\infty}^{+\infty} F_{i} \delta(t-i)
$$

where $\left\{F_{i}\right\}$ is any dynamical time series produced by a map $f$ :

$$
F_{i+1}=f\left(F_{i}\right)
$$

To evaluate the energy integral (Eq.3), we consider first the effect of a single pulse at $t=t_{0}$ on $R$. Energy input, $R_{0}$, by this pulse is:

$$
\begin{gathered}
R_{0} \equiv \int F \delta\left(t-t_{0}\right) d x(t)=\int_{t_{0}-0}^{t_{0}+0} F \delta\left(t-t_{0}\right) \frac{d x}{d t} d t \\
=F \cdot\left(\left.\frac{d x}{d t}\right|_{t=t_{0}-0}+\left.\frac{d x}{d t}\right|_{t=t_{0}+0}\right) / 2 .
\end{gathered}
$$

By integrating Eq.(1) between $t=t_{0}-0$ and $t=t_{0}+0$, we obtain through Eq.(9):

$$
R_{0}=F \cdot\left(2 v_{0}+F / m\right) / 2,
$$

where $\left.v_{0} \equiv \frac{d x}{d t}\right|_{t=t_{0}-0}$. In case that the relaxation time of the velocity, $m / \gamma$, is smaller than the unit interval of the pulse, $m / \gamma \ll 1$, the effect of the velocity, $v_{0}$, on $R_{0}$ is negligible. As we will consider the overdumping motion, we will neglect the effect of $v_{0}$. Average input energy per unit time is obtained using an invariant density of the map:

$$
\langle R\rangle \simeq\left\langle\frac{F_{i}^{2}}{2 m}\right\rangle=\frac{1}{2 m} \int F^{2} \rho(F) d F,
$$

where $\rho(F)$ is the invariant density of the map. The average displacement, $\langle\Delta x\rangle$, in the unit time is:

$$
\langle\Delta x\rangle=P_{t r} \cdot 2 L
$$

where $P_{t r}$ is a probability that a particle crosses a peak of the periodic potential per unit time. We call $P_{t r}$ as "barrier crossing probability" hereafter that is obtained in a theory of chaotic noise [8]. Inserting Eq.(12) into Eq.(河), we obtain the average coherent energy, $\left\langle E_{l}\right\rangle$, that the system acquires in a unit time:

$$
\left\langle E_{l}\right\rangle=P_{t r} \cdot 2 L \cdot l .
$$

Inserting Eqs.(11) and (13) into Eq.(5), we obtain the average efficiency of the energy transformation:

$$
\left\langle\epsilon_{t}\right\rangle=\frac{l \cdot P_{t r} \cdot 2 L}{\frac{1}{2 m} \int F^{2} \rho(F) d F} .
$$

In the derivation of the efficiency, we have introduced some approximations. Thus, we will compare this analytical results with numerical ones. For demonstration, we use tent map chaos [9] as a dynamical noise. We set:

$$
F_{i+1}=1 / 2-2\left|F_{i}\right| .
$$

The tent map has a constant invariant density and $\delta$ correlation function, of which the simple statistical character is useful for an analysis. In Fig.1, we show the typical dependence of the efficiency of energy transformation on the strength of the load. The numerical result shows that the efficiency monotonically increases up to $l \sim 10^{-2}$; and after the peak, the efficiency decreases suddenly. The present theory sufficiently predicts the increase of the efficiency up to the peak. The numerical result shows that decrease of the efficiency starts faster than that of theory. This disagreement is attributed mainly to the disagreement of the barrier crossing probability, $P_{t r}$, that is estimated by a previous theory [8]: Before the efficiency starts saturation, the system crosses the potential barrier unidirectionally. In this regime, the theory of chaotic noise 8 precisely predicts the barrier crossing probability, $P_{t r}$. However, when the saturation begins, the system crosses it in both directions. In this regime, the theory of chaotic noise does not precisely predicts the barrier crossing probability; the disagreement of the efficiency emerges in this regime.

In case that the potential barrier is $60 \%$ less than that of Fig.1, the efficiency of energy transformation (Fig.2) is higher than that of Fig.1. Although the order of energy transformation of Fig.2 differs from Fig.1, the functional forms of the two Figures are almost the same. The difference of the maximum efficiencies of energy transformation between Fig.1 and Fig.2 is attributed to the difference of the barrier crossing probabilities. 
FIG. 1. Efficiency of energy transformation of a system where $m=0.1, \gamma=1.0, L=5.0$ and $h=0.5$.
FIG. 2. Efficiency of energy transformation of a system where $m=0.1, \gamma=1.0, L=2.0$ and $h=0.2$.

In this paper, we have derived an energetics of a ratchet system that transforms dynamical noise to coherent energy. The formula of the efficiency of energy transformation itself would be certainly robust as far as overdumping condition, $m / \gamma \ll 1$ holds: the formula (Eq.14) is not restricted to special shape of the potential. The precision of the efficiency much depends on the precision of the barrier crossing probability, $P_{t r}$.

From the viewpoint of dynamical systems, one can imagine that the efficiency should reflect the complexity of the noise. The complexity can be estimated by, for example, the Kolmogorov Entropy 10]. We conjecture that high efficiency would emerge when the Kolmogorov Entropy of the dynamical noise is low. If the functional form (Eq.8) of the noise is restricted, we can find an realization of this conjecture [11]. We are now at the starting point of this analysis.

Real protein motors is known to operate at surprisingly high efficiency of energy transformation 12. However, none of the ratchet models have not successfully explained this high efficiency yet, because energetics had not been derived in ratchet models until Ref. [6] except for Ref. [2], to my knowledge. We have not studied to optimize the efficiency by varying the shape of the potential and parameters of the system. Further study of the optimal efficiency of ratchet models will bring us a new insight as to the biologically possible mechanism of protein motors.

We gratefully acknowledge K. Sekimoto, Y. Sawada, S. Nasuno, S. Sasa, T. Tsuzuki for helpful comments and discussions and also acknowledge T. Fujieda and F. Takagi for critical reading of the paper. Numerical calculation was partly performed by the workstations of YITP, Kyoto University. This work is supported in part by the Japanese Grant-in-Aid for Science Research Fund from the Ministry of Education, Science and Culture (No. 09740301).

[1] Early works where the idea of noise-induced transport was discussed are found in M. Büttiker, Z. Phys. B 68, 161 (1987); R. Landauer, J. Stat. Phys. 53233 (1988).

[2] Efficiency of energy transformation was first discussed in a ratchet model of molecular motor: R. D. Vale and F. Oosawa, Adv. Biophys. 26, 97 (1990). Because the efficiency derived in this paper was based on the incorrect calculation by Feynman [1], the efficiency was estimated much higher than that of Ref. 6].

[3] For examples: M. O. Magnasco, Phys. Rev. Lett. 71, 1477 (1993); J. Rousselet, L. Salomé, A. Ajdari, and J. Prost, Nature 370, 446 (1994); A. Ajdari, D. Muramel, L. Peliti, and J. Prost, J. Phys. I (France) 4, 1551 (1994); C. R. Doering, W. Horsthemke, and J. Riordan, Phys. Rev. Lett. 72, 2984 (1994). R. D. Astumian and M. Bier, Phys. Rev. Lett. 72, 1776 (1994); L. Faucheux, L. Bourdieu, P. Kaplan, and A. Libchaber, Phys. Rev. Lett. 74, 1504 (1995).

[4] T. Hondou, J. Phys. Soc. Jpn. 63, 2014 (1994); T. Hondou and Y. Sawada, Phys. Rev. Lett. 75, 3269 (1995); ibid. 76, 1005 (1996).

[5] R. P. Feynman, R. B. Leighton, and M. Sands, The Feynman Lectures in Physics (Addison-Wesley, Massachussets, 1966).

[6] K. Sekimoto, J. Phys. Soc. Jpn. 66, 1234 (1997).

[7] See, for example, C. W. Gardiner, Handbook of Stochastic Methods 2nd ed. (Springer-Verlag, Berlin, 1990).

[8] Formulae of barrier crossing probability, $P_{t r}$, for a generalized map are given in T. Hondou and Y. Sawada, Phys. Rev. E54, 3149 (1996). 
[9] S. Grossmann and H. Fujisaka, Phys. Rev. A26, 1779 (1982).

[10] See, for example, H. G. Schuster, Deterministic chaos, (Physik-Verlag, Berlin, 1984).

[11] T. Hondou, (unpublished).

[12] For example, R. C. Woledge, N. A. Curtin and E. Homsher, Energetic Aspect of Muscle Contraction (Academic Press, New York, 1985). 